\title{
Keyword Search over Distributed Graphs with Compressed Signature
}

\author{
Srihari Ch, A.Manasa, K.Venkataramana, G.Pavani
}

\begin{abstract}
Catchphrase search graph has attracted tons research interest, due to the fact the version diagram can speak pleasant for maximum prepared and dependent database and scan the slogan can launch good sized statistics to the customer with out simple data about the sample and language questions. Practically speaking, information photographs may be very huge, for instance, Web-scale diagram containing billions of vertices. The fine in elegance technique utilizing delivered collectively for the calculation of the slogan seek process diagram, after which they do now not deserve to chart a totally huge, because of confined computing power and further area at the server focused. To remedy this hassle, we look at the slogan test graph scale web page is introduced in splendid situation. We first offer calculation effortlessly believe the response request productive questions. In any case, the calculation of flood searching harmless make use of search techniques that obtain huge time and system overhead. To treatment this weak point, we're at that time advise pursuing calculation based totally marks. In precise, we construct that encodes vertex signatures short way an excellent manner from factor to some random catchphrase in the graph. Thus, we can locate solutions to questions by investigating the dearth of way, with the aim that point and correspondence low value. In addition, we changed the diagram facts in the organization after dividing irregular underlying with the goal that the method is primarily based at the sign greater interesting. Finally, the results of our trial show achievability of our proposed method in carrying out watchword top view diagram statistics Web scale.
\end{abstract}

Keywords:Keyword search, Search problems, Algorithm design and analysis, Servers, Partitioning algorithms, Distributed databases, Resource description framework

\section{INTRODUCTION}

Mark based definitely pruning is significantly cited as a a hit method to decorate query execution of diagram format coordinating on general named charts. Maximum structures which use signature-put together pruning case its advantages with understand to all datasets and inquiries. Be that as it may, the viability of mark based pruning modifications rather amongst diverse RDF datasets and profoundly identified with their dataset attributes. We see that the presentation income thru signature-put together pruning rely now not simply with respect to the dimensions of the RDF diagrams, but further at the vital chart form and the multifaceted nature of inquiries. This spurs us to advocate an adaptable RDF thinking shape, known as RDF- $\square$, which

Srihari Ch, Professor, Department of CSE, Siddhartha Institute of Technology \& Sciences, Narapally, Ghatkesar, Hyderabad, Telangana, India

A.Manasa, Assist. Prof, Department of CSE, Siddhartha Institute of Technology \& Sciences, Narapally, Ghatkesar, Hyderabad, Telangana, India.

K.Venkataramana, Assocate. Prof, Department of CSE, QISIT, Ongole, (Email: ramanakaveripakam@gmail.com)

G.Pavani, Assist. Prof, Department of CSE, Siddhartha Institute of Technology \& Sciences, Narapally, Ghatkesar, Hyderabad, Telangana, India.
Revised Manuscript Received on 14 September, 2019.

particularly uses signature-primarily based definitely pruning by way of assessing the attributes of RDF datasets and inquiry formats. We show off the versatility and productiveness of RDF- $\square$ by means of exploratory consequences the use of every real and engineered datasetswe study the viability of mark based totally pruning for thinking diagram organized facts making use of chart formats that specialize in diagram prepared RDF information.Neighborhood signature-based totally pruning has been applied broadly to beautify execution of diagram layout coordinating (in view of sub chart isomorphism) trouble. A sizable variety of types of neighborhood signature documents have been created [15-20]. Even as signaturebased totally pruning can be high-quality for inquiries on RDF datasets, there are some variables to be considered. To begin with, RDF charts have great hub marks, and use URIs to distinguish a exquisite many property which can be usually lengthy strings, and incompletely entered catchphrases are applied in determining RDF question formats. On this way, the form of neighborhood facts and the approach of community regulation check in inquiry getting ready need to help inquiries with fractional watchwords. Moreover, viability of mark prepare pruning profoundly relies upon with recognize to the qualities of datasets and questions [7]. The primary diagram form of RDF datasets can go from extreme social like shape to subjective charts for various applications. Finally, signaturebased completely pruning need to be utilized specifically for diverse RDF charts and questions. We center spherical three question assessment standards for RDF charts, in particular,

(1) Flexibility and expressiveness of inquiry codecs: question layouts which are adaptable to indicate the 2 catchphrases and the diagram shape; (2) utilization of the characteristics of dataset and query codecs for inquiry development; (three) Scalable inquiry evaluation with a purpose to scale to RDF datasets with at the least heaps and hundreds triples.

\section{II.METHODOLOGY}

\section{Catchphrase search on charts}

Watchword are searching for over a chart famous a substructure of the diagram containing all or a portion of the data catchphrases. A massive part of past strategies here discover related negligible bushes that spread all of the query catchphrases. As of late, it is been demonstrated that discovering subgraphs in region of timber can be an increasing number of useful and useful for the customers. Nonetheless, the prevailing tree or diagram based totally 
totally strategies can also create replies wherein some substance hubs (i.E., hubs that incorporate enter watchwords) are not near every different. Also, at the same time as scanning for solutions, those techniques may additionally look into the whole diagram as opposed to just the substance hubs. This could additionally spark off horrible displaying in execution time. To cope with the above troubles, we endorse the difficulty of coming across $r$ inner circles in charts. A r-faction is a meeting of substance hubs that unfold all the information catchphrases and the separation amongst each hubs isn't exactly or equivalent to r. A precise calculation is suggested that discovers all rcoteries within the information diagram. Likewise, a guess calculation that produces r-clubs with 2-estimation in polynomial postponement is proposed. Huge execution thinks approximately making use of huge actual informational indexes confirm the effectiveness and exactness of coming across r-inner circles in diagrams.

Catchphrase searching for, an high-quality thing for enhancing important facts from a number of facts, has as of late been examine for keeping apart information from prepared statistics. Prepared facts are usually displayed as diagrams. For instance, considering IDREF/identity as connections, XML files can be displayed as charts. Social databases can likewise be tested utilizing charts, wherein tuples are hubs of the diagram and far flung key connections are edges that interface two hubs (tuples) to each other [6, 12]. In such fashions, watchword seek assumes a key task in locating precious facts for the clients. Customers mostly do not have ok statistics approximately the shape of information.

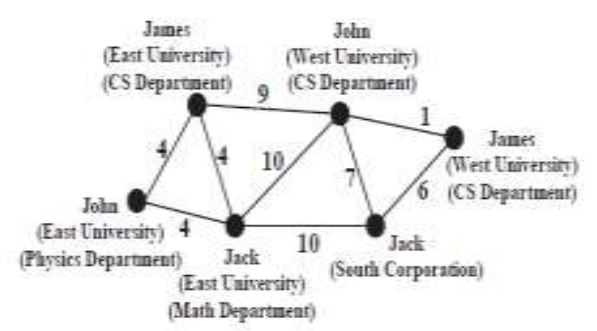

Figure 1: A sample graph. The shortest distance between a pair of nodes is shown on their edge.
Tree-based totally strategies produce snappy solutions. All the more as of late, the approach delivers a chart that is proposed, which offers an more and more instructive solution. Nonetheless, the tree or diagram primarily based method has the accompanying issues. To start with, at the same time as some substance hubs within the tree or diagram created close to one another, there is probably content material hubs within the results which might be a protracted way from each other, which means that a feeble dating between's the hub substance may also exist in the timber is discovered or chart. We contend that, accepting each one of the watchwords which can be similarly widespread, the effects of which incorporate solid relationship (eg, short separations) among each pair of hubs content should be higher on people who incorporate a feeble relationship. Second, the method for the existing diagram or tree by using investigating the substance and non-content hub in the chart whilst searching results. Since there might be lots or maybe a large variety of hubs within the diagram input, this technique makes a few excessive recollections and reminiscence unpredictability. In this paper, we advocate to find out r-click-click on as any other way to address the problem of a watchword search. A rmembership is a lot of hubs content contains all info watchwords and the most limited separation between every pair of hubs isn't always extra outstanding than $r$. Advantages of discovering r-click on-click on is as according to the subsequent. To start with, the r-click on all of the substance hub fits close to one another (as an instance, inner a separation $r$ ). Besides, there's no compelling reason to analyze the entirety of the hubs inside the diagram input when observed r-click on-snap of the privilege if the file is manufactured. This decreases the inquiry space through collapsing. 


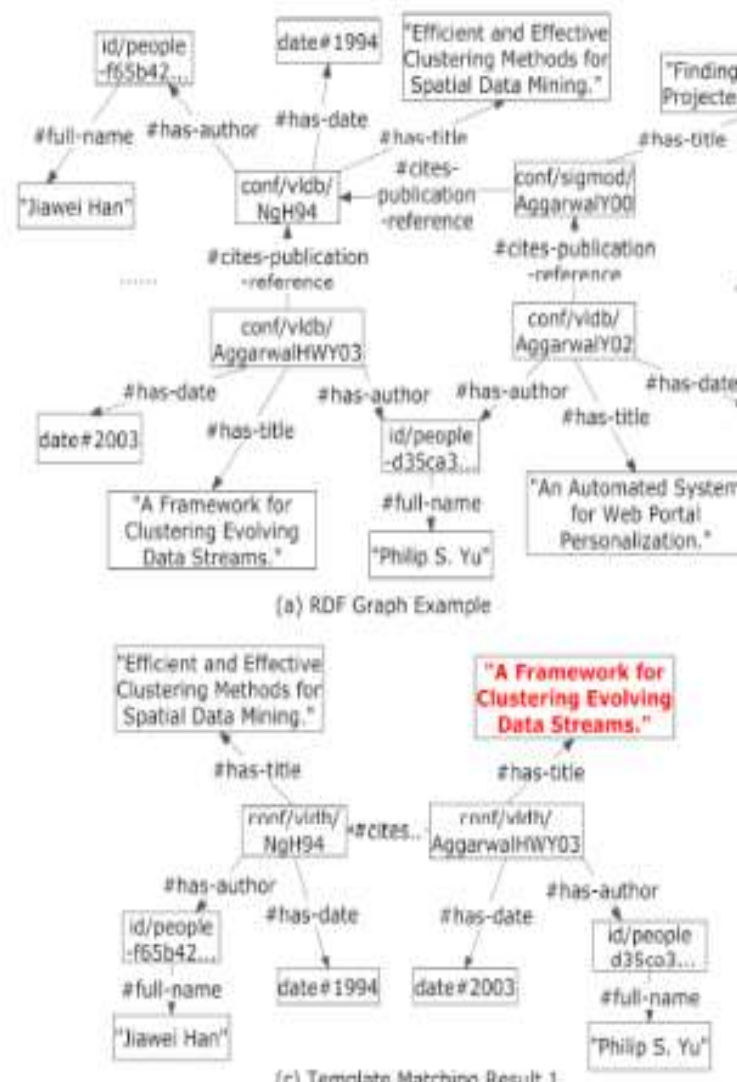

(c) Template Matching Result 1

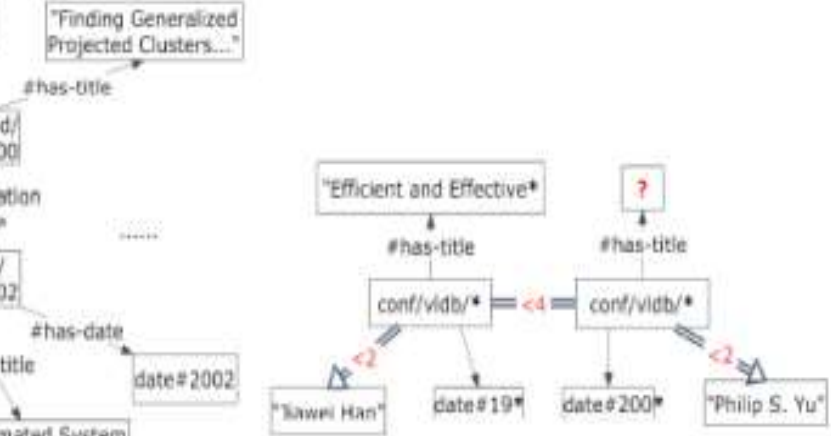

(b) Graph Template Example

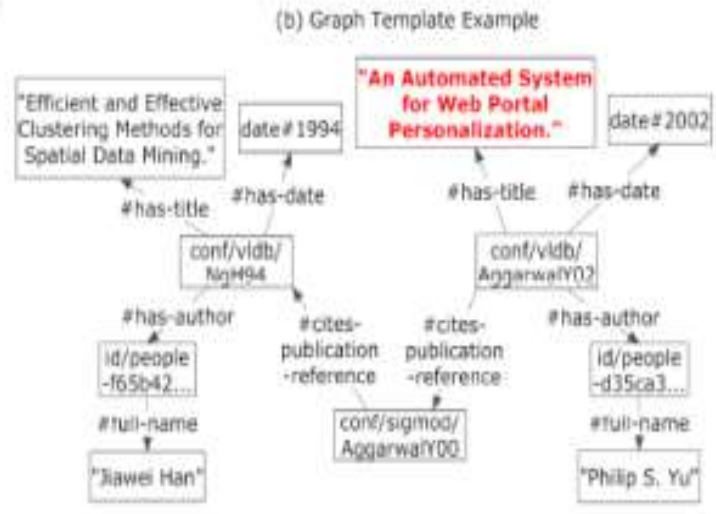

(d) Template Matching Result 2 .

Fig. 1. RDF Graph and Query Template with Matching Results

\section{Template Matching for RDF graphs}

Here we characterize chart layout coordinating for RDF diagrams with an adaptable inquiry layout helping ways, separation necessities, and incomplete coordinating of catchphrases:

A RDF Graph is a coordinated chart $\mathrm{G}=\mathrm{V}, \mathrm{E}, \mathrm{L}, \mathrm{F}$ in which $\mathrm{V}$ is lots of hubs speakme to subjects, objects or each. $\mathrm{E} \subseteq \mathrm{VxV}$ is lots of coordinated edges speakme to predicates indicating from topics gadgets. $\mathrm{L}$ is a mark set for topics, objects and predicates. F: V/E $\rightarrow$ L suggests the mapping capacity among hubs/edges to labels.A affiliation aspect (E $\Leftrightarrow)$ speaks to a way wij among hubs WIJ and ni, where nj can be one directional or bi-directional. Articulation $\square$ depicts the separation imperatives of (separation is the length of the maximum short manner between hubs).

An affiliation area (e $\Leftrightarrow)$ speaks to a way $\omega$ ij amongst hubs wij and wij, wherein wij can be one directional or bidirectional. Articulation $\square$ portrays the separation limitations of wij (separation is the period of the briefest way among hubs).

\section{Related paintings}

The extra part of the techniques to cope with watchword searching for over diagrams discover timber as answers2. In, a retrogressive quest calculation for handing over Steiner trees is displayed. A effective programming approach for discovering Steiner timber in charts are exhibited in. Notwithstanding the reality that the dynamic programming method has exponential time multifaceted nature, it's far sensible for enter questions with modest variety of catchphrases. In, the creators proposed calculations that produce Steinertrees with polynomial deferral. The calculations pursue the Lawler's technique. Because of the NP-fulfillment of the Steiner tree issue, developing timber with unmistakable roots are furnished recently. Squints improve crafted with the aid of the usage of a efficient ordering shape.

There are techniques that discover sub charts in region of trees for catchphrase are trying to find over diagrams. The number one method discovers r-variety Steiner charts that consist of everything of the info catchphrases. For the reason that calculation for discovering r-span charts report them paying little heed to the statistics watchwords, if part of the profoundly placed r-range Steiner diagrams are remembered for other larger charts, this device may also omit them. What's more, it is able to create copy and repetitive effects. The 2 nd methodfinds multi-focused sub charts, known as networks. In every community, there are somecenter hubs. There exists at any rate a solitary manner amongst each middle hub and each substance hub with the stop goal that the separation is not exactly Rmax. Parameter Rmax isused to manipulate the dimensions of the network. 
The authorsof propose a calculation that can offer all networks in a self-assertive request and some different calculation that produces placed networks in polynomial postponement. The position of a community is based upon on the bottom an incentive the numerous all out location masses from one of the focuses to the whole thing of the substance hubs. Coming across com-munities as the reaction for watchword searching for over diagram records has 3 issues. Even as part of the substance hubs can be near each different, the others might not. Additionally, for locating each community, the calculation considers everything of the hubs internal Rmax pinnacle techniques from every substance hub as a possibility for a middle hub. This activates terrible run-time execution. At closing, even as which includes focus and amongst interfere hubs within the right responses can locate the connections some of the substance hubs, these center and transitional hubs might be superfluous to the inquiry, which makes some answers hard to decipher. Our proposed model improves the network method through the use of (1) discovering r-factions in which all of the substance hubs are close to every other, (2) improving the run-time by using investigating simply the substance hubs at some stage in search, and (three) lessening the superfluous hubs through turning in a Steiner tree (in place of a diagram) to discover the connection a number of the substance hubs in a r-inner circle.

\section{LITERATURE SURVEY}

Coming across pinnacle-ok Min-value connected trees in Databases

Bolin Ding, Jeffrey Xu Yu, Shan Wan, Lu Qin, Xiao Zhang, Xuemin Lin

It's miles broadly understood that the joining of database and statistics restoration strategies will grant clients with a wide scope of notable administrations. On this paper, we have a look at dealing with a l-watchword inquiry, p1, p2, • $\mathrm{pl}$, against a social database which may be displayed as a weighted diagram, $\mathrm{G}(\mathrm{V}, \mathrm{E})$. Right here $\mathrm{V}$ is a lot of hubs (tuples) and $\mathrm{E}$ is a lot of edges speakme to outside key references between tuples. Let $\mathrm{Vi} \subseteq \mathrm{V}$ be a number of hubs that include the watchword pi . We check discovering pinnacle-adequate least charge related timber that contain at any price one hub in every subset $\mathrm{Vi}$, and mean our undertaking as GST-k. At the point while ok $=1$, it's miles called a base value bunch Steiner tree trouble this is whole. We see that the amount of catchphrases, 1, is little, and endorse a unique parameterized arrangement, with 1 as a parameter, to locate the ideal GST-1, in time intricacy $\mathrm{O}(3 \mathrm{ln}$ $+2((1+\log n) n+m))$, in which $n$ and $m$ are the quantities of hubs and edges in chart G. Our solution can address diagrams with infinite hubs. Our GST-1 arrangement may be efficaciously reached out to assist GST-ok, which beats the contemporary GST-k arrangements over each weighted undirected/coordinated diagrams. We led extensive trial research, and record our finding.

Squints: Ranked key-word Searches on Graphs

Hao He, Haxtun Wang, Jun Yang, Philip S. Yu

Question managing over chart prepared facts is getting a rate out of a developing type of uses. A top-ok watchword search inquiry on a chart nds the pinnacle ok solutions as indicated by way of using some positioning requirements, wherein each answer is a substructure of the diagram containing all query catchphrases. Present day techniques for supporting such inquiries on brand new diagrams experience the sick results of some downsides, e.G., bad maximum pessimistic scenario execution, no longer exploiting records, and high reminiscence requirements. To cope with those troubles, we advocate BLINKS, a bi-degree ordering and question dealing with plan for pinnacle-ok watchword are seeking on charts. Flickers pursues a pursuit technique with provable execution limits, at the same time as moreover abusing a bi-degree document for pruning and quickening the quest. To decrease the list vicinity, BLINKS parcels an information chart into hinders: The bilevel document shops rundown statistics at the square degree to start and guide are seeking for amongst squares, and steadily aspect via point statistics for each square to quicken search inner squares. Our trials show that BLINKS gives orders-ofsize execution development over existing methodologies.

Catchphrase primarily based Tweet Extraction and Detection of related subjects

Amrutha Bennya, Mintu Philipb

As in line with the studies of man or woman to man or woman communication locations in 2013 , seventy $3 \%$ of on line grown-united statesare currently utilising in any occasion one of the lengthy variety casual verbal exchange locales and out of this 40 two $\%$ make use of incredible systems management sites10. From the ones examinations, the significance of man or woman to character conversation locales in our everyday regular life is extremely smooth. Lengthy range interpersonal verbal exchange locations have been at the beginning utilized by humans actually to speak with partners thru messages. Be that as it may, presently the project of prolonged range informal verbal exchange places in the information spreading place is a non-beside the point one. A large detail of lengthy range interpersonal communique destinations is that they supply a degree that interfaces. People from numerous portions of the arena, alongside the ones strains spreading the records swiftly. A part of the first-rate long variety interpersonal conversation locales are fb, Twitter, Integra, LinkedIn, and so on.

In the direction of gold standard Graph seek techniques In this section, we take a look at the pursuit approach of BLINKS on a full-size stage and evaluation it subjectively and past strategies.

In opposite search with none listing that might deliver diagram community statistics beyond a solitary jump, we can answer the question with the resource of investigating the chart starting from the hubs containing at any fee one inquiry keywordsuch hubs can be identied effectively via a converted rundown report. This technique typically activates a retrogressive pursuit calculation, which fills in as pursues.

1. Whenever during the retrogressive pursuit, allow Ei imply the affiliation of hubs that we recognise can arrive at inquiry watchword ki; we name Ei the organization for ki. 2. At the beginning, Ei starts offevolved due to the fact the arrangement of hubs Oi that legitimately consist of ki; we do not forget this underlying set the agency reason and its

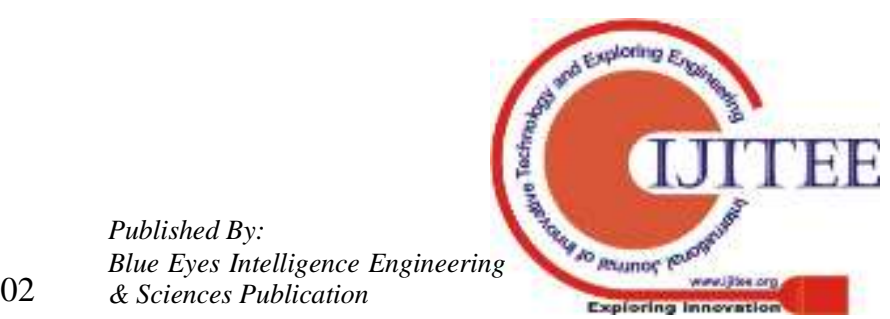


element hubs catchphrase hubs. 3. In every seek step, we pick an drawing close component to 1 in every of lately visited hubs (nation v), and in a while pursue that edge in opposite to visit its deliver hub (country u); any Ei containing $\mathrm{v}$ currently extends to encompass u too. When a hub is visited, all its coming near edges end up recognized to the quest and reachable for choice by way of way of a future enhance. Four. We have determined a solution root $\mathrm{x}$ if, for every bunch $\mathrm{Ei}$, both $\mathrm{x} \in \mathrm{Ei}$ or $\mathrm{x}$ has an side to $3 \mathrm{hub}$ in Ei. The rst in contrary watchword seek calculation modified into proposed with the useful resource of Bhalotia et al. [3]. Their calculation makes use of the accompanying two methodologies for choosing what to visit straightaway. For accommodations, we dene the great methods from a hub $\mathrm{n}$ to a selection of hubs $\mathrm{N}$ due to the fact the briefest true methods from $\mathrm{n}$ to any hub in $\mathrm{N}$.

Equi-separation improvement in each institution: This system chooses which hub to go to for extending a catchphrase. Instinctively, the calculation extends a bunch through the usage of visiting hubs prepared via increasing top strategies from the corporation inception. Formally, the $\mathrm{v}$ in opposite, for some $\mathrm{v} \in \mathrm{Ei}$ ) is the hub with the most limited separation (among all hubs now not in Ei) to Oi.

Separation adjusted extension crosswise over bunches: This technique chooses the outskirts of which catchphrase will be extended. Instinctively, the calculation endeavors to modify the separation among every bunch's root to its outskirts over all businesses. Enormously, allow (u, Ei) be the hub bunch pair to such an volume that $\mathrm{u} 6 \in \mathrm{Ei}$ and the best approaches from $\mathrm{u}$ to $\mathrm{Oi}$ is the most short possible. The bunch to increase next is Ei. Bhalotia et al. [3] didn't talk approximately the optimality of the over two structures. Right here, we provide, as a ways as we ought to probable realize, the relaxation thorough examination of their optimality. In the first place, we set up the optimality of equidistance extension internal every clus.
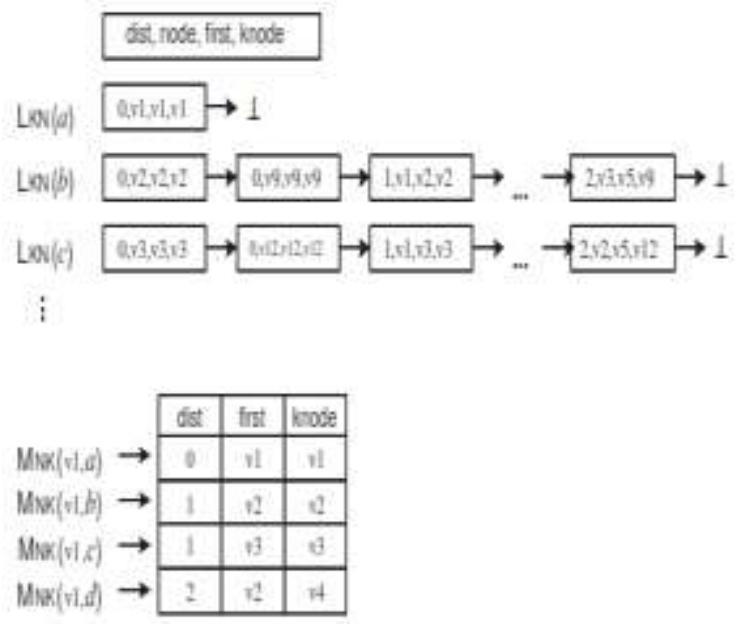

$$
\text { : }
$$

\section{Figure Keyword-node lists and node-keyword map.}

\section{A Single-Level Index}

Notion and Index shape For each bunch Ei, the stan-dard technique for executing equi-separation in opposite extension is to keep up a want line of hubs asked through their suitable procedures from watchword ki. The road speaks to a "boondocks" in investigating ki, which also can hub $\mathrm{u}$ to visit next for bunch Ei (through following part $\mathrm{u} \rightarrow$

broaden exponentially in size in any event, for scanty charts. The time multifaceted nature is moreover excessive, as it takes $\mathrm{O}(\log \mathrm{n})$ time to locate the most increased need hub, in which $\mathrm{n}$ is the dimensions of the line. We are able to probable lower the lifestyles multifaceted nature of search.

A standard manner to cope with enhance online execution is to in step with-form some disconnected calculation. We pre-decide, for each watchword, the maximum quick accurate ways from each hub to the catchphrase (or, all of the more sincerely, to any hub containing this watchword) inside the statistics diagram. The very last results is an series of catchphrase hub statistics. For a catchphrase w, LKN (w) indicates the rundown of hubs that can arrive at watchword w, and those hubs are asked by their separations to w. Every passage in the rundown has four fields (dist., hub, first, knode), wherein dist. Is the maximum confined separation amongst hub and a hub containing w; hub is a hub containing $\mathrm{w}$ for which this briefest separation is stated; first is the primary hub on the briefest way from hub to knode. 1 In determine 4, we deliver some quantities of the catchphrase hub information worked for the diagram in parent 1 (accepting all edges have weight 1). For instance, within the rundown for catchphrase $b$, the number one phase is $(0, v 2, v 2, v 2)$, which re bits the manner that v2 can arrive at the watchword $b$ with dis-daze zero and first and hub happen to be v2 itself. The last passage (2, v3, v5, v9) mirrors the manner that the most quick way from $v 3$ to $b$ is $\mathrm{v} 3 \rightarrow \mathrm{v} 5 \rightarrow \mathrm{v} 9$ with separation 2 .

\section{Report creation}

The single-stage list may be populated by way of in contrary developing hunts starting from catchphrases. To check within the separations amongst hubs and watchwords, we concurrently run $\mathrm{N}$ duplicates of Dijkstra's unmarried supply maximum short way calculation in a regressive developing layout, one for every one of the $\mathrm{N}$ hubs in the chart. This way is just like the catchphrase query calculation given via BANKS [3], on the other hand, in reality we're creating a document rather than noting on the net inquiries. We exclude the point thru point calculation proper here. Note that the time multifaceted nature of this calculation is $\mathrm{O}(\mathrm{N} 2)$, it really is excessive for large charts. Our effects in phase five moreover diminish this multifaceted nature.

The single-diploma report may be applied for any scoring functionality with specific root and match-distributive semantics. Anyways, the listing development calculation delineated above moreover assume the chart separation semantics (cf. Place 2).

\section{Lists}

We use two lists to be specific, IDMap and NI (community meantime) Indexes, to help effective assessment of fractional catchphrases and association edges.

1. The arrangement of IDs for all RDF marks shapes an period in-between of back to lower lower back numbers;

2. IDs of marks are allocated in lexicographic request.

IDMap Index basically maps RDF marks into complete range IDs in lexicographic request. For fractional 
catchphrases indicated as prefixes of RDF marks, the appearance-into time is $0(\log \mathrm{N})$, wherein $\mathrm{N}$ is the complete extensive variety of RDF names, due to the reality every unmarried coordinating identification shape one period inbetween of decrease lower back to again entire numbers. Unique files can likewise be evolved to quicken appearance time of incomplete watchwords.

\section{IV.DISCUSSIONS \& RESULTS}

The NI report is built depending on the IDMap document thru gathering the marks (IDs) of buddies of each hub into identification interims. It's miles set up as a desk with five segments: for any hub ni $\in \mathrm{G}$, it consists of identification of ni, Distance, Label identification meantime, wide variety of filed neighbor hubs in this passage, and neighbor hub IDs. The gap is the length of the most restrained manner from ni to the ordered neighbor hub. The first-class (bad) separation shows that the filed hub is a forward (in contrary) neighbor. There are two predefined parameters for the NI report: firstclass filed separation dmax and binning element $M$. The neighbor hubs having a similar separation are assembled, ordere with the aid of their IDs, and parceled into columns by way of the binning aspect $\mathrm{m}$, which constrains the finest style of filed neighbor hubs in every report passage. Increasing the maximum extreme filed separation dmax appreciably builds the distance of NI file with the aid of along side more listed friends for every hub. Be that as it may, large dmax implies increasingly more community statistics recorded which improves each the pruning strength and the machine time for association edges with lengthy separation necessities. NI report is supposed to be quality when fractional catchphrases are indicated as prefixes of RDF hub marks. NI document can be visible as a widespread type of the marks applied in each GraphQL [17] and SPath [18]. NI listing furthermore offers powerful evaluation to association edges as multi-bounces pals are filed through using using the hub IDs.

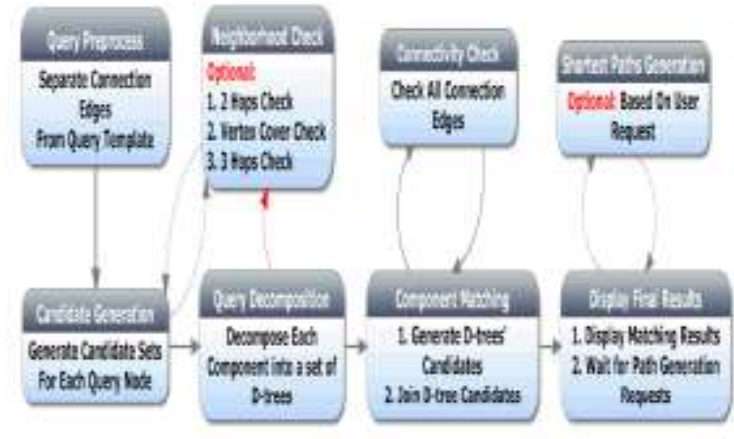

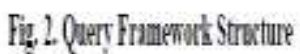

\section{RDF Query Framework}

RDF tool call for [Figure 2] making use of D-Tree1 as inquiry unit premise. This form begins through isolating the threshold of the association call for layouts, which could bring about a format with particular associated segments. IDMap listing is then used to locate an appropriate opportunity for each hub a solicitation to analyze the incomplete catchphrase. Check manner state of affairs at that point specifically picked to reduce the association of possibility for each hub query. Each element is associated decayed right into a development of D-timber. Every single reasonable possibility for every deteriorating $\mathrm{D}$-tree is produced, and afterward consolidated to have a counterpart for each segment. Thing association is at prolonged remaining prepared using NI document to supply the ultimate suit. In light of customer request, facet areInstantiated establishments through figuring out all the maximum constrained way.

\section{Ecological Containment test}

Test the detainment condition for NI document is characterised as the intervening time identity test

(here, we assume midway watchword set as a prefix of a hub mark) .Keywords, units of qualities as distance, take a look at (overall appearances within the Distance) for every fractional catchphrase saved up for each hub inquiry. Appears at conveyed by using the usage of watchword community fractional for my part, and the amount of occasions of each catchphrase incomplete perception. The expression "one in every of a kind contained" inside the that means of 3.1 method that the hub $\square \square$ can't be carried out to coordinate more than one period in-between identification. On the off chance that one catchphrase consists of the watchword fractional incomplete one-of-a-kind, matched rely esteem is refreshed. Calculation 1 suggests the ecological method exams, distance, bear in mind units related to the solicitation hub and pi qj fractional watchword is meant with the aid of wij

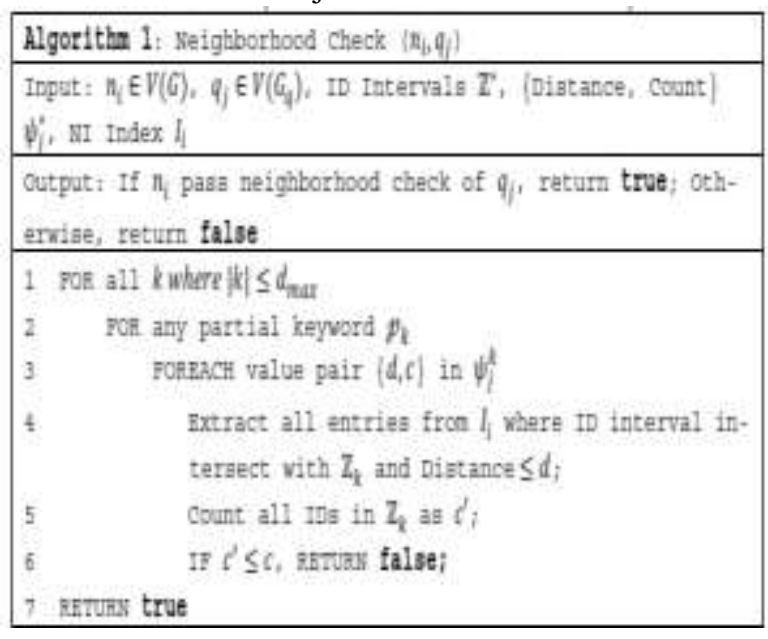

Test situation greater nice for the watchword midway: 1) just the record sections with mark identity hose met with the period in-between id fractional catchphrase must be taken; 2) all the identification in the listing passage that suits the watchword applies to in component if the identification period in-between includes an incomplete catchphrase Label identity interim.

\section{Coordinating aspect}

Facet can interface the element request or be part of two particular segments. Inside the event that the threshold is in one of the components, at that point check the network is carried out to trim the planned segment. If not, test the supply is applied to determine if the 2 up-and-comers segments may be joined or no longer. For interior edge of

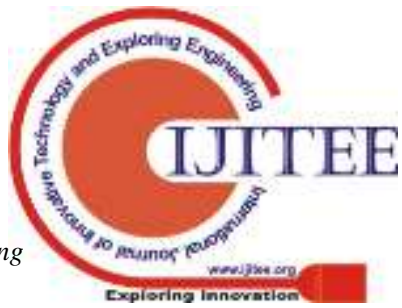


the element associations, the amount of community take a look at is really the size of the competitor set of segments. To the threshold of the connection between the segments, the amount of availability take a look at is predicated upon the end result of the dimensions of the planned element set. In the maximum pessimistic situation, in the occasion that we've got an association of segment $\mathrm{N}$ may be joined via the threshold of the affiliation, the amount of assessments network should be completed may be as massive as in an effort to enhance inquiry execution, guidelines are utilized to determine the request for the association manner the rims: 1) among segment part associations are dealt with in advance than intra-vicinity component institutions; 2) intralocation phase affiliation littlest gadgets prepared in the request for contender for the essential set.

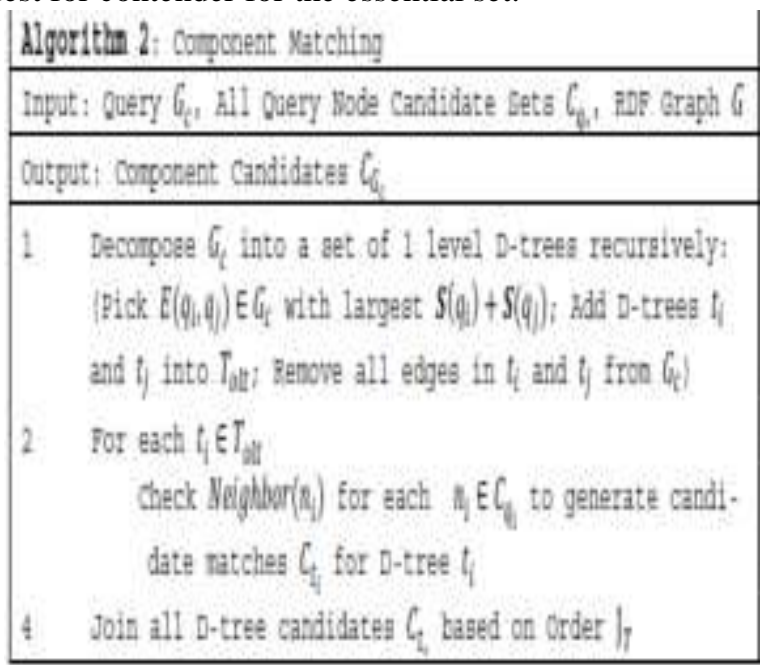

A Take a look at surroundings optimized for the keyword partial: 1) excellent the index entries with label identification hose intersected with the interval identity partial key-word wants to be taken; 2) all of the id within the index access that suits the important thing-word applies to in component if the identification c language consists of a partial key-word Label identification c language.

\section{Matching factor}

Component can be part of the detail demand or be a part of first rate additives. If the brink is in one of the additives then test the connectivity is used to trim the possible issue. If not, take a look at the connectivity is used to determine whether or not or now not the 2 applicants additives may be joined or not. For the interior fringe of the factor connections, the number of connectivity check is exactly the dimensions of the candidate set of components. To the threshold of the connection among the components, the variety of connectivity check depends at the fabricated from the size of the viable element set. In the worst case, if we have a sequence of aspect $\mathrm{N}$ may be joined by using the threshold of the relationship, the quantity of exams connectivity wishes to be performed may be as huge as with a view to improve query overall performance, policies are used to decide the order of the relationship system the rims: 1) inter-factor part connections are processed earlier than intra-aspect component connections; 2) intra-part trouble connection smallest merchandise processed in the order of applicants for the number one set.

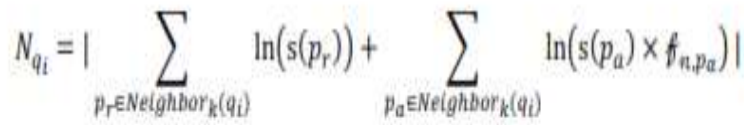

Wherein pr is any relationship predicate, dad is any assets predicate, good enough is the amount of jumps of network take a look at system, $\mathrm{n}$ is the length of fractional catchphrases related with place dad, $\mathrm{S}(\mathrm{pr})$ and $\mathrm{S}(\mathrm{pa})$ are predicate selectivity for Pr and dad, and fn, dad is exacting selectivity

\section{$R D F-h$}

RDF-h calculation streamlines the query execution of an inquiry format via specifically utilizing mark prepare pruning based totally with appreciate to 2 situations: (I) regardless of whether or not, for this question layout, the amount of center of the road competition and joins can be diminished; and (ii) whether or not or now not the community structure of an inquiry layout has excessive selectivity to provide effective pruning. On the off risk that the 2 situations are valid, the mark based totally pruning is utilized in making geared up the query layout. After inquiry disintegration (discern 2), the quantity of up-and-comer creating cycles for each D-tree and the assessed amount of middle of the street tree joins are checked with new release Threshold T1 and be part of Threshold T2. At the off threat that any variety surpasses the brink, at that thing community check is taken into consideration. The subsequent degree is to sign in the community Selectivity for all query hubs to test if there can be any inquiry hub with community Selectivity arriving at the insignificant Selectivity Threshold T3. Assuming this is the case, network check is used. The choices of limits T1, T2 and T3 effect the inquiry execution of the RDF-h; and their functions can be tuned by the use of utilising an example set of question formats at the given dataset. Subtleties of the tuning approach is available in [28].

\section{Space comparison for unique Indexes}

The regular area required for NI listing ismaximum ordered separation, is the binning issue, $\delta$ is the amount of hubs within the RDF diagram and $\square$ is the ordinary hub degree. Figure three indicates information on various files for numerous RDF datasets in stage of the primary dataset. For all great NI documents, the binning aspect $\mathrm{m}$ is about as 5. Identity Map list in all fairness little contrasted and NI information. Four specific NI facts are thought approximately proper here, and are utilized by diverse questioning techniques (1 jump listing for "STWIG+", 2 leap file for "Spat(NI2)" and " $\square-2$ Hops", three jump files for " $\square-3$ Hops" and vertex unfold listing for " $\square-\mathrm{VC} ")$. Glaringly, the space required for NI lists increments as increments. The vertex spread record uses 2 soar buddies for hubs within the vertex spread, and 1 leap pals for distinct hubs. Therefore, the space necessity for the vertex spread report is amongst 1 leap list and 2 jump list. The everyday hub diploma of the RDF chart likewise impacts report sizes. 


\section{DBLP dataset}

Diagram era We to start with produce a hub marked coordinated chart from the DBLP XML facts (http://dblp.Uni-trier.De/xml/). The first XML data is a tree in which every paper is a hint sub tree. To make it a diagram, we embody kinds of non-tree edges. First of all, we companion papers through references. 2nd, we make a similar author underneath various papers percent an ordinary hub. The chart is large, however for the most element it's miles as yet a tree and now not especially intriguing for diagram seek. To function the motive for diagram are seeking for, we make it extra chart like thru (1) evacuating components in every paper that are not exciting to watchword seek, as an instance, url, ee, and so forth.; (2) expelling most papers not referencing distinct papers, or now not being referenced with the useful resource of different dad pers. At long very last, we get a chart containing $50 \mathrm{~K}$ papers, $409 \mathrm{~K}$ hubs, $591 \mathrm{~K}$ edges, and $60 \mathrm{~K}$ unique decrease-cased keywords.As appeared in parent , BLINKS beats the Bidirectional pursuit by means of at any charge a request for greatness by way of and large, which suggests the adequacy of the bi-stage record. It moreover indicates that the quantity of watchword hubs (see desk 1) isn't the absolute maximum sizeable problem influencing response time. For example, despite the truth that the two watchwords in Q6 show up in scarcely any hubs, the Bidirectional hunt makes use of greater time on Q6 than Q1 Q5. There are in reality often predominant elements, but they can't be statically quantized through the amount of watchword hubs. First is the dimensions of the outskirts prolonged at some point of the inquiry. The quantity of watchword hubs simply decides the underlying boondocks. Whilst an outskirts arrives at hubs with giant in-levels, the size of the need line increments substantially. Q6 has a place with this example. Second is the thing at which we're able to securely forestall are seeking for. It profoundly relies upon pruning adequacy, which is predicated upon the character of the correct responses acquired up till this factor. For instance, Q6 has just one answer (the inspiration element dblp), so no pruning sure (the score of the k-th answer) may be set up to surrender are seeking for early.

The other belief is that no matter the reality that an appropriate inquiry area of a query relies upon numerous factors, inquiries containing increasingly more catchphrases will in fashionable have larger hunt regions as each watchword has its very own outskirts. Within the trial, Q1Q6 each contain watchwords, Q7-Q8 3, and Q9-Q10 4. The aftereffects of BLINKS display longer reaction time for inquiries with extra catchphrases. We furthermore analyze BLINKS using one in all a kind parceling calculations. Almost always, BFS-based dividing shows desired execution over METIS-primarily based one. Be that as it may, it isn't always in every case actual, appeared inside the following studies on the IMDB dataset. We likewise watch the impact of rectangular size. For the maximum detail talking, with bigger squares, searches will consist of plenty less cursors. Be that as it is able to, for the same inquiry, the query time of larger square dividing would not typically beat that of littler one. In fact, the question time is stimulated with the aid of using severa considered one of a type variables, as an example, the stacking of rectangular files, the manner in which the pursuit vicinity traverses squares, and so on.Index overall performance Now we look at the impact of rectangular size the ordering execution of BLINKS, as an extended way as ordering time, variety of entryways, and list period, which is probably seemed in discern, for my part. Every parent indicates effects under vet diverse designs. The preliminary four range of their normal rectangular sizes (one hundred, three hundred, six hundred, and one thousand) and all usage METIS-based totally parceling. The final one has one thousand and utilizations the more trustworthy BFS-based parceling.

Determine shows apportioning time and ordering time for every format. The initial 4 arrangements have similar dividing time, that's ruled through the METIS calculation, on the same time as ordering time increments reliably at the same time as squares turn out to be bigger. This expanding sample is likewise seen in discern eight(c), wherein larger squares motive more document passages. The last setup (1000BFS) ap-employs the essential BFS-primarily based dividing, so it desires significantly much less apportioning time. However, interestingly, it likewise takes extensively much less ordering

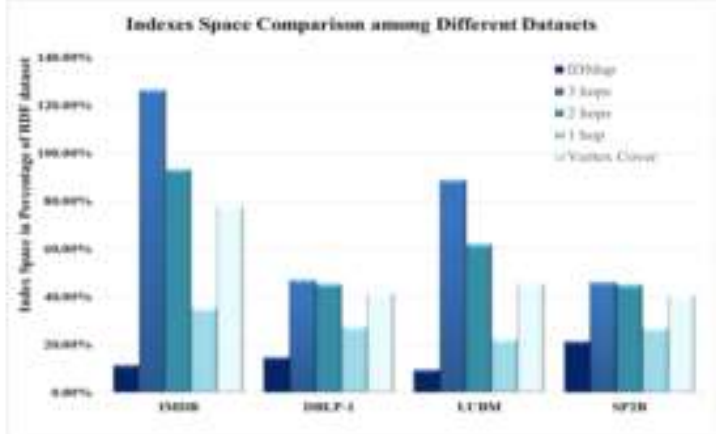

Fig. Indexes Space Comparison

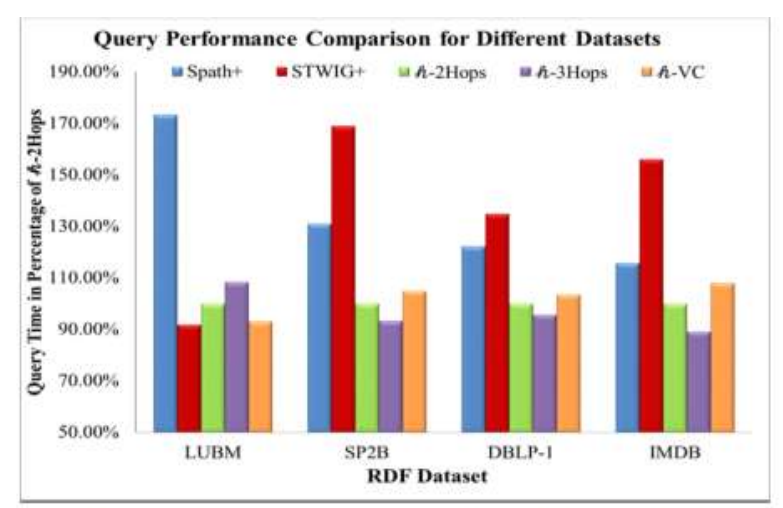

Fig. Query Comparison (Datasets) 


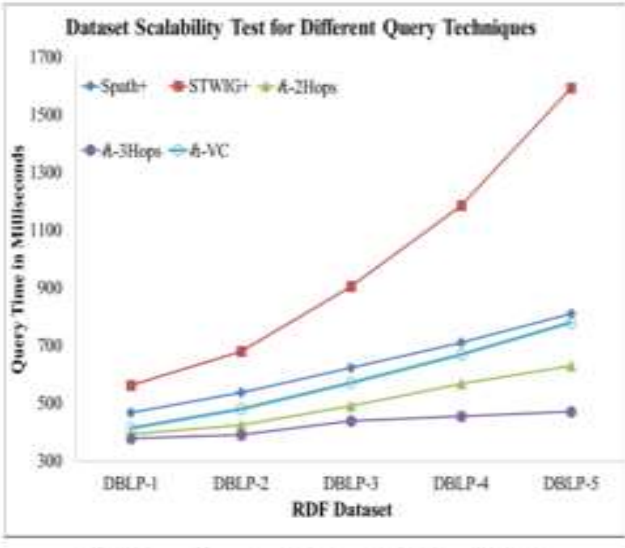

Fig. Dataset Scalability Test

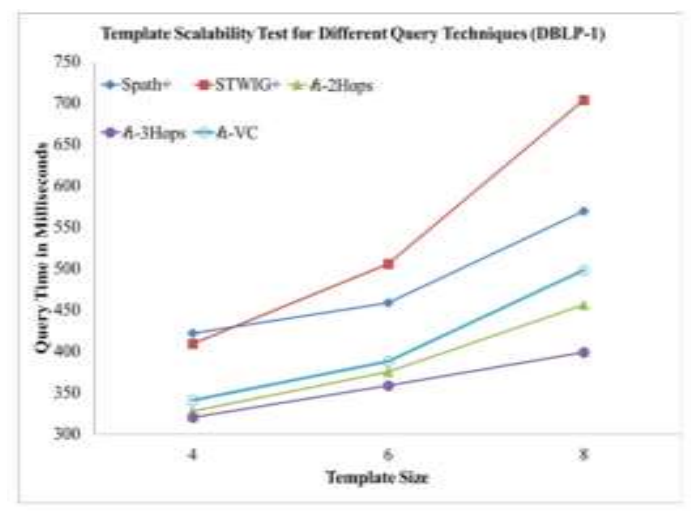

Fig. Template Scalability Test

SP2B and DBLP every hub ha ordinary degree of approximately three, and it is approximately eight to LUBM and IMDB. For that reason, the rise in NI report area with extra leap ordered the buddies plenty extra organized to LUBM and IMDB contrast and SP2B and DBLP.

\section{Questions performance for exceptional Datasets}

For each dataset, forty questions had been abnormal in duration 6 created independently. Consequences of the implementation of all strategic questions inquiry for more than one datasets that appear in discern 4. To LUBM, neighborhood exams worthless to maximum questions due to the structure of easy diagrams and signs of a good uniform. Because of the intensity of close by assessments aren't massive pruning, the leap of corrupt environmental exams for LUBM exhibition due to the time overhead. Three different RDF datasets, SP2B, DBLP and IMDB has a design execution as each unique. Proper to form, excessive close by assessments plum midway up-and-comers and be part of good enough, and, consequently, greater leap of environmental tests carry a higher execution. Because Späth (Ni2) the usage of more environmental assessments, conduct of investigations to languish due to the extra LUBM, however the advantage with a sturdy pruning for exclusive datasets. On the upside, as STWIG + by no means use additional environmental tests, it performs properly for LUBM, but skilled competition half of vain dataset created for SP2B, DBLP and IMDB. RDF- $\square$ calculation beat both Späth (Ni2) and STWIG + given that becoming a member of alternatives. Essential questions treated legally via

opposition D-tree age and joined while complex queries expanded through the use of greater environmental exams. Checking 2 and 3 bounces neighbor jumped greater appealing in contrast IMDB and SP2B and DBLP given that 2 way and three way leap jumping rarely prompts a greater modest huge form of requests hub artificial with 2 or three buddies soar bounce. Comparison and $\square-2$ Hops, functionVC $\square$ top notch due to the fact the space required is littler. Implementation $\square-2$ Hops rather extra regrettable than $\square$ 2Hops, proper to form.

\section{Programs for coupling DFS / BFS to restore combinatorial}

Shows and overhead of past plans hesitation determined to venture squares problem (eg NUGENT 221). This software program executes a calculation department certain: recursively producing hub in pursuit tree, which has a hub and a maximum depth of 221938 22.Locally, each processor actualized as a rely of direction fseq consecutive calculations that actualize a depth first seek (DFS) in tree , Empower for reminiscence backup and similarly to enhance the developing of trees without duplicates (the children of the hub $\mathrm{n}$ successively product of the expected $\mathrm{n}$ with backtracking). To limit the time this is crucial, parallel computation choice actualize fpar first are looking for (BFS) calculations extent. At the point while the processor receives inactive, it took the maximum skilled hub of non-inert arbitrarily choose the processor (Extract Par). This parallel calculation introduces overhead for the tests brought about the iCluster22 copy.The hub, a collection of 104 hubs connected by using 100Mbps Ethernet set. Every hub highlight two Itanium-2 processor $(900 \mathrm{MHz})$ and $3 \mathrm{~GB}$ of reminiscence close by. The calculation is parallelized using Kaapi. Stage parallelism (restrict) may be balanced: after a positive intensity, sub-tree of the hub processed regionally by means of fseq. This boundary is marked easy granularity and should be picked as an awful lot as the extent that the calculation of a close-by clock fseq is sort of same with the parallelism overhead time.

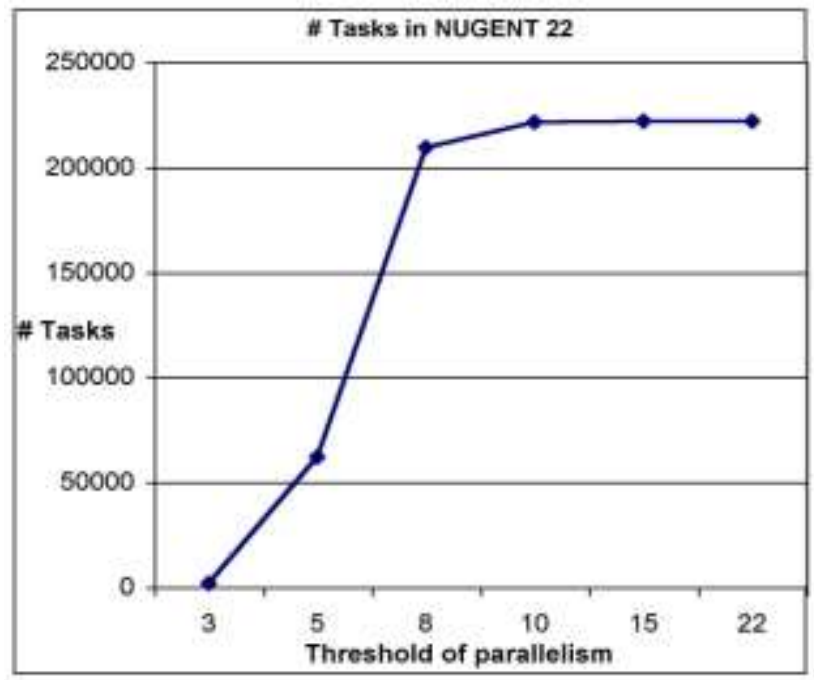

Impact of granularity 


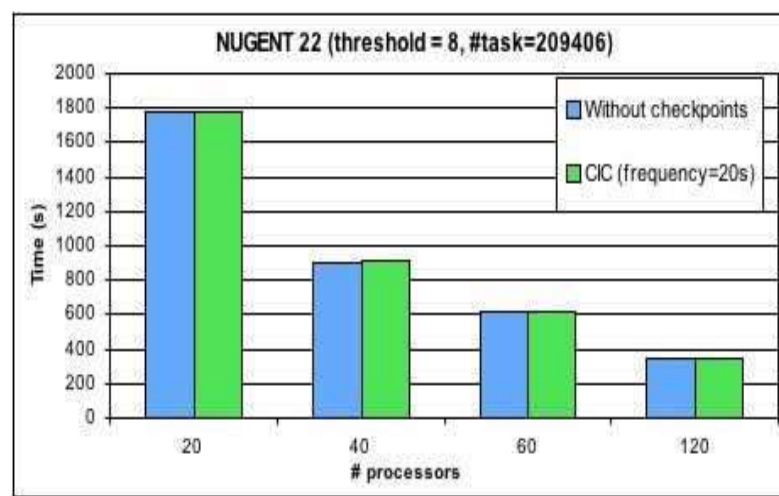

Execution time (sequential time: 34,695 s)

\section{CONCLUSIONS}

In this paper, we determine the viability of mark put together pruning with recognize to wondering diagram organized RDF records utilizing chart layouts. Because of the perception that mark based totally pruning isn't always continuously precious, we endorse a $1 / 2$ and half of calculation RDF- $\square$, which especially makes use of neighborhood check depending on the attributes of RDF datasets and inquiry layouts. By tuning the parameters, RDF- $\square$ calculation can consequently seize go to consumer inquiry designs and be modified in accordance with increase the benefits of mark based totally pruning which offer a general $30 \%$ question execution development for abnormal created subgraph inquiries. In mild of the RDF dataset attributes examination, we will likewise distinguish datasets as for the everyday degree of execution profits from signature-primarily based pruning. There are some bearings for broadening this work. Improving the adaptability of our machine to an awful lot bigger datasets is full-size. This present day paper's method scales to RDF datasets with a wonderful many triples that is sensibly large, but there are real datasets with billions of triples. Without appropriate stress approaches, one can barely bring together the NI document on billion hub charts. A few inquiries can wind up with quite large number of suits, and a first rate positioning capability is must had been equipped to restore the most important outcomes in rank request.

\section{REFERENCES}

1. Michael A. Drinking spree, Erik D. Demaine and Martin FarachColton. Reserve negligent b-trees. SIAM J. Register., 35(2):341-358, 2005.

2. Michael A. Drinking spree, Jeremy T. Fineman, Seth Gilbert, and Bradley C. Kuszmaul.Concurrent reserve negligent b-bushes. In SPAA'05: Proceedings of the 17th yearly ACMsymposium on Parallelism in calculations and designs, pages 228-237, New York, NY, USA, 2005. ACM Press.

3. R.S. Winged animal. Rationale of Programming and Calculi of Discrete Design, part Introduction to the Theory of Lists. Springer-Verlag, 1987.

4. M. Cole. Parallel Programming with List Homomorphism's. Parallel Processing Letters,5(2):191204, 1995

5. El-Mostafa Daoudi, Thierry Gautier, Aicha Kerfali, R'emi Revire, and Jean-Louis Roch.Algorithmes parallels 'grain adaptatif et programs. Method etScience Informatiques, 24:1-20, 2005.
6. F. D'Azevedo and J. Dungaree. The plan and execution of the parallel out-of-center scalapack lu, qr and cholesky factorization schedules. Specialized Report CS-ninety seven-347, University of Tenessee, january 1997. Http://www.Netlib.Org.

7. Jean-Guillaume Dumas. Effective dab item over restricted fields. In Victor G. Ganzha, Ernst W. Mayr, and Evgenii V. Vorozhtsov, editors, Proceedings of the seventh In-ternational Workshop on Computer Algebra in Scientific Computing, Yalta, Ukraine, pages 139-154. Technische Universit“at Mu“nchen, Germany, July 2004.

8. Jean-Guillaume Dumas, Thierry Gautier, and ClementParent. Limited Field Linear Algebra Subroutines. In Teo Mora, supervisor, Proceedings of the 2002 International Symposium on Symbolicand Algebraic Computation, Lille, France, pages sixty three74. ACM Press, New York, July 2002.

9. Jean-Guillaume Dumas, Pascal Giorgi, and ClementParent. FFPACK: Finite Field Linear Algebra Package. In Jaime Gutierrez, manager, Proceedings of the 2004 International Symposium on Symbolic and Algebraic Computation, Santander, Spain, pages119126. ACM Press, New York, July 2004.

10. Matteo Frigo and Steven G. Johnson. The structure and execution of FFTW3.Proceedings of the IEEE, ninety three(2), 2005. Extraordinary issue on "Program Generation, Optimization, and Adaptation". 